\title{
A two-stage inexact programming with value-at- risk for water resources management
}

\author{
Xiangming Kong ${ }^{*}$, Donglin Wang, Chunxiao Wang, Yu Wang, Xiufeng Yao, and Xiaoming \\ Liu \\ Beijing Polytechnic, Beijing 100176, China
}

\begin{abstract}
In this study, the application of a two-stage inexact programming with value-at-risk (TIPV) model in water resources system planning has been developed. The TIPV method is intended to tackle the inexact parameters and the risks of economic loss. The application of case study shows that more alternatives under multiple levels of risks could be generated. The amount of water shortages and the width of system benefit intervals would decrease as the risk increases. TIPV could provide more effective information for stakeholders to recognize social policies with maximized system benefits under various risk levels.
\end{abstract}

\section{Introduction}

In water-resources system, many components and parameters are influenced by uncertainties. For example, the economic parameters may be represented by fuzzy numbers or intervals. And the stream conditions may exhibit stochastic uncertainties. These uncertainties should be tackled by appropriate inexact optimization methods. Maqsood et al.[1] proposed a method for water resources system planning by combing interval and fuzzy uncertainties into a two-stage stochastic programming. A new multi-purpose method has been presented by Khatiri et al.[2] in order to reach a compromise among multiple decision makers through identifying hydro-environmental management and policies. In this study, a two-stage inexact programming with value-at-risk (TIPV) model would be proposed for revealing dual uncertainties and reflecting the risks of recourse. A case study of TIPV on water resources management would be an attempt to combine the value-at-risk $(\mathrm{VaR})$, the fuzzy boundary intervals and the random variables into a general two-stage programming framework.

\section{Two-stage Inexact Programming with VaR}

A two-stage inexact programming with VaR (TIPV) model is developed by introducing VaR into a two-stage inexact programming. TIPV can reveal uncertainties expressed intervals and probabilities in objective function and constraints. Moreover, possible risk loss of net system benefit could be measured.

\footnotetext{
*Corresponding author: 101582@bpi.edu.cn
} 


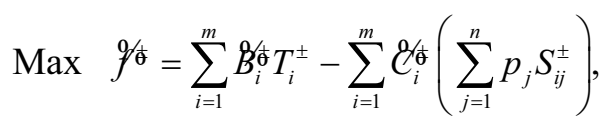

s.t.

$$
\begin{aligned}
& \sum_{i=1}^{m}\left(T_{i}^{ \pm}-S_{i j}^{ \pm}\right) \leq q_{j}^{ \pm}, \quad \forall j, \\
& \xi_{F V a R}^{ \pm}\left(\sum_{i=1}^{m} \mathcal{C}_{i}^{\bar{\theta}}\left(\sum_{j=1}^{n} p_{j} S_{i j}^{ \pm}\right), \beta\right) \leq \tau, \\
& S_{i j}^{ \pm} \leq T_{i}^{ \pm} \leq T_{i \max }^{ \pm}, \quad \forall i, j, \\
& S_{i j}^{ \pm} \geq 0, \quad \forall i, j .
\end{aligned}
$$

Where $f^{\frac{0}{\hat{\theta}}}$ is system benefit represented by a fuzzy boundary interval; $B_{i}^{\bar{\phi}}$ and $\mathcal{C}_{i}^{\bar{\phi}}$ are benefit and penalty, respectively; $T_{i}^{ \pm}$is water allocation in first-stage; $S_{i j}^{ \pm}$is water shortage in second-stage; $q_{j}^{ \pm}$is available water resources under probability $p_{j} ; \tau$ is maximum acceptable loss under probability $\beta ; T_{i \max }^{ \pm}$is maximum allowable water allocation; $i=1,2, \ldots, m$ is index for water users; $j=1,2, \ldots, n$ is index for probabilities.

For decision makers, water resources could be promised to multiple demanders based on the requirement and the expected amounts of water resources in the first-stage. However, the inexact amounts of steam flow may bring out the water shortage problems. Therefore, economic penalties usually happen in the second-stage. TIPV is an attempt to combine the value-at-risk, fuzzy boundary intervals and random variables into a general two-stage programming framework. Economic penalties in the second-stage could be qualified and controlled by the VaR constraint in TIPV. That is to say, the optimal water resources allocations could be generated through maximum system benefits under controllable economic penalties.

The solution algorithm of TIPV could be developed based on two-step and VaR transformation methods. The two-step method is a useful method for solving two-stage programming[3]. Therefore, the difficulty of solution algorithm is the transformation of VaR constraint. Let $\stackrel{0}{0}$ be intervals with bounds of trapezoidal fuzzy random variable. The inexact VaR function $\xi_{F V a R}^{ \pm}\left(\ell \frac{ \pm}{0}, \beta\right)$ are formulated as[3,4]:

$$
\xi_{\text {FVaR }}^{ \pm}(\stackrel{\mathscr{V}}{\mathrm{o}}, \beta)= \begin{cases}a_{\xi}^{ \pm}+2\left(b_{\xi}^{ \pm}-a_{\xi}^{ \pm}\right) \beta, & \text { if } \beta \leq 0.5, \\ 2 c_{\xi}^{ \pm}-d_{\xi}^{ \pm}+2\left(d_{\xi}^{ \pm}-c_{\xi}^{ \pm}\right) \beta, & \text { if } \beta>0.5 .\end{cases}
$$

\section{Application in Water Resources Management}

A case study on water resources management has been illustrated for application of the proposed TIPV method. There is an unregulated reservoir in this study. It supplies water resources to three water users (i.e. a municipality, an agricultural sector and an industry concern). Table 1 gives the economic data, including benefit and penalty. Table 2 gives the stream flow data. The related data about water allocation targets is shown in Table 3. 
Table 1. Economic data $\left(\$ / \mathrm{m}^{3}\right)$.

\begin{tabular}{lll}
\hline & Net benefit $B_{i}^{\circ}$ & Penalty $\mathscr{C}_{i}^{\phi}$ \\
\hline Municipal $(i=1)$ & {$[(83,86,89,92),(101,104,107,110)]$} & {$[(211,215,219,223),(270,273,276,279)]$} \\
Industrial $(i=2)$ & {$[(40,45,50,55),(65,70,75,80)]$} & {$[(55,58,61,64),(84,87,90,93)]$} \\
Agricultural $(i=3)$ & {$[(21,22,23,24),(28,29,30,31)]$} & {$[(43,46,49,52),(65,68,71,74)]$} \\
\hline \multicolumn{3}{c}{ Table 2. Stream flows. } \\
\hline Flow level & Probability (\%) & Stream flow $q_{j}^{ \pm}\left(\right.$in $\left.10^{6} \mathrm{~m}^{3}\right)$ \\
\hline Low $(\mathrm{L})(j=1)$ & 0.3 & {$[3.4,4.2]$} \\
Medium $(\mathrm{M})(j=2)$ & 0.4 & {$[7.7,12.6]$} \\
High $(\mathrm{H})(j=3)$ & 0.3 & {$[15.0,18.6]$} \\
\hline
\end{tabular}

Table 3. Water allocation targets $\left(10^{6} \mathrm{~m}^{3}\right)$.

\begin{tabular}{llll} 
& $\begin{array}{l}\text { Municipal } \\
(i=1)\end{array}$ & $\begin{array}{l}\text { Industrial } \\
(i=2)\end{array}$ & $\begin{array}{l}\text { Agricultural } \\
(i=3)\end{array}$ \\
\hline Water allocation target $X_{i}^{ \pm}$ & {$[1.5,2.3]$} & {$[2.7,3.6]$} & {$[3.0,5.2]$} \\
Maximum allowable allocation $X_{i \max }^{ \pm}$ & 8.0 & 8.0 & 8.0 \\
\hline
\end{tabular}

Table 4 gives the solutions obtained from TIPV. There exist water shortages for industrial and agricultural water users under low level of stream flow. Only agricultural water user's water demand could not be satisfied under medium level of stream flow. And under high level of stream flow, all water demand from three water users could be satisfied. Moreover, the amount of water shortage decreases as the risk level increases. It means that the VaR constraint for penalty has an effective on risk management.

Table 4. Solutions obtained from TIPV $\left(10^{6} \mathrm{~m}^{3}\right)$.

\begin{tabular}{llllllllll}
\hline \multirow{2}{*}{ Risk level $\beta$} & \multicolumn{2}{l}{ Shortage $Y_{i j}^{ \pm}$} \\
\cline { 2 - 10 } & $Y_{11}^{ \pm}$ & $Y_{21}^{ \pm}$ & $Y_{31}^{ \pm}$ & $Y_{12}^{ \pm}$ & $Y_{22}^{ \pm}$ & $Y_{32}^{ \pm}$ & $Y_{13}^{ \pm}$ & $Y_{23}^{ \pm}$ & $Y_{33}^{ \pm}$ \\
\hline 0.5 & 0 & {$[1.70,2.50]$} & 5.11 & 0 & 0 & {$[0,3.31]$} & 0 & 0 & 0 \\
\hline 0.7 & 0 & {$[1.70,2.50]$} & 4.54 & 0 & 0 & {$[0,2.74]$} & 0 & 0 & 0 \\
\hline 0.9 & 0 & {$[1.70,2.50]$} & 4.39 & 0 & 0 & {$[0,2.59]$} & 0 & 0 & 0 \\
0.95 & 0 & {$[1.70,2.50]$} & 4.35 & 0 & 0 & {$[0,2.54]$} & 0 & 0 & 0 \\
\hline
\end{tabular}

Table 5 gives the results of system benefits under various risk levels. It indicates that the lower bound of system benefit increases as the risk level increases, and the upper bound of system benefit decreases as the risk level increases. That is to say, the increasing risk level would lead to an decreasing of interval width.

Table 5. Net system benefit $\left(\$ 10^{6}\right)$.

\begin{tabular}{ll}
\hline Risk level $\beta$ & Distribution of net system benefit $f^{0 / 6}$ \\
\hline 0.5 & {$[(193.46,227.98,231.83,251.02),(515.30,539.18,563.06,586.94)]$} \\
\hline 0.7 & {$[(207.47,255.21,260.26,280.65),(506.71,530.53,554.36,578.18)]$} \\
\hline 0.9 & {$[(211.24,262.54,267.91,288.62),(504.51,528.32,552.13,575.94)]$} \\
\hline 0.95 & {$[(212.32,264.63,270.10,212.32),(503.98,527.78,551.59,575.39)]$} \\
\hline
\end{tabular}

Figure 1 shows the optimal water allocation targets under $\beta=0.5,0.7,0.9$ and 0.95 . There always exist water shortage for water user of agriculture section under different stream flow levels under each risk levels. That is because the net benefit from agriculture section is the lowest among three water users. Moreover, comparing with other water users, the penalty for agriculture section is the lowest. Figure 2 shows the lower bound of optimal 
water allocation amounts. Figure 3 shows the corresponding upper bound. Results could supply multiple strategies for decision makers in water resources system planning.

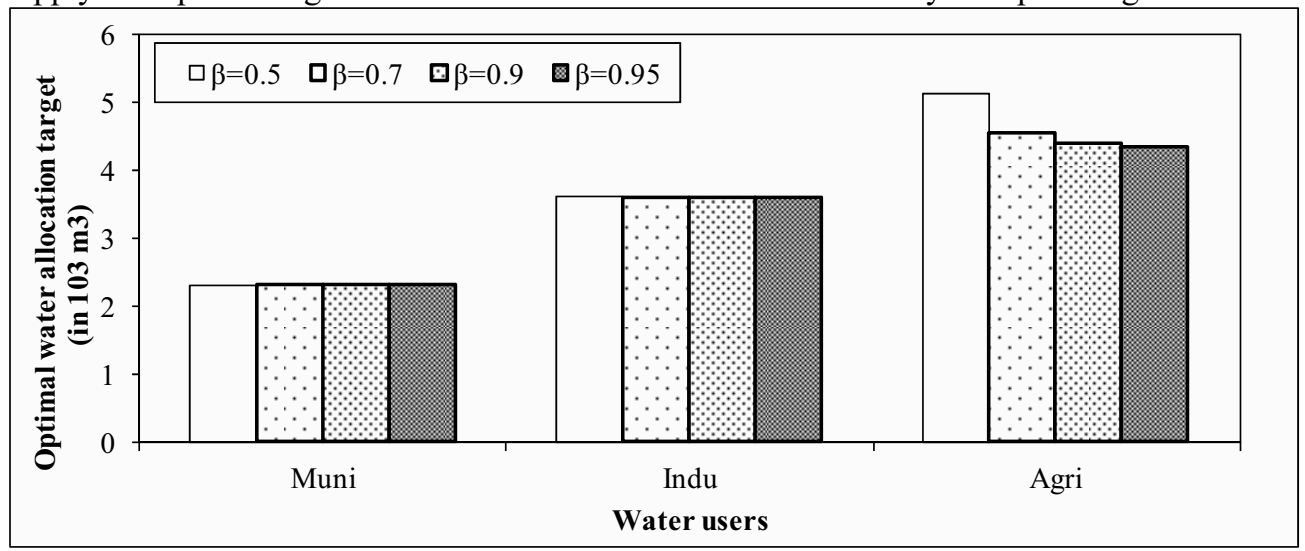

Fig. 1. Optimal water allocation targets.

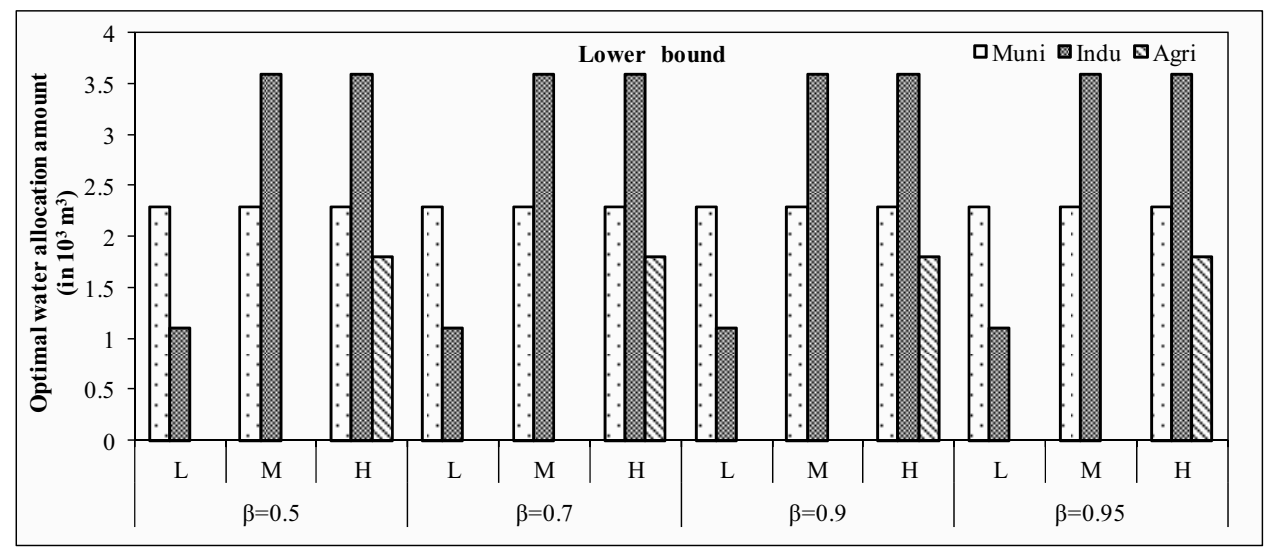

Fig. 2. Lower bound of the optimal water allocation amount.

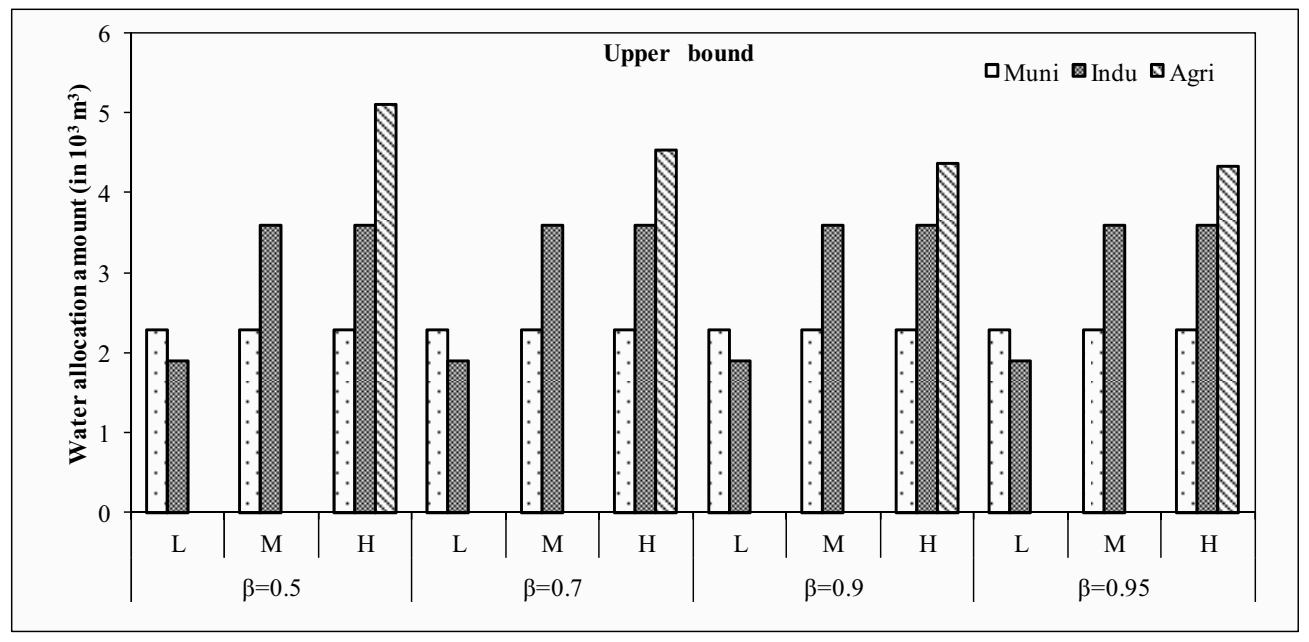

Fig. 3. Upper bound of the optimal water allocation amount. 


\section{Concluding Remarks}

A case study on water resources management has been illustrated for application of a twostage inexact programming with VaR (TIPV) method in this study. The advantages of TIPV are that (1) it could deal with inexact parameters presented as fuzzy boundary intervals and probability distributions; (2) it could reveal the potential risk of recourse at the second-stage. The results of case study show that more alternatives under various risk levels could be generated. The width of system benefit intervals would decrease as the risk level increases. Once it appears water shortage, the promised water allocation to agriculture section would be reduced because of the lowest net benefit and penalty of agriculture section among three water users. However, the amount of water shortage would decrease as the risk level increases. It means that the VaR constraint for penalty has an effective on risk management.

\section{References}

1. Maqsood I., Huang G. H., Yeomans J. S. (2005). An interval-parameter fuzzy twostage stochastic program for water resources management under uncertainty. European Journal of Operational Research, 167(1):208-225. http://doi.org/10.1016/j.ejor.2003.08.068

2. Khatiri K. N., Niksokhan M. H., Sarang A., Kamali A. (2020). Coupled simulationoptimization model for the management of groundwater resources by considering uncertainty and conflict resolution. Water Resources Management, 34(11):3585-3608. http://doi.org/10.1007/s11269-020-02637-x

3. Kong X. M., Huang G. H., Fan Y. R., Li Y. P., Zeng X. T., Zhu Y. (2017). Risk analysis for water resources management under dual uncertainties through factorial analysis and fuzzy random value-at-risk. Stochastic Environmental Research \& Risk Assessment, 31:2265-2280. http://doi.org/ 10.1007/s00477-017-1382-3

4. Shiraz R.K., Tavana M., Fukuyama H. (2020). A random-fuzzy portfolio selection DEA model using value-at-risk and conditional value-at-risk criteria. Soft Computing, 24:17167-17186. http://doi.org/10.1007/s00500-020-05010-7 